\title{
ON THE SINGULARITIES OF GEGENBAUER (ULTRASPHERICAL) EXPANSIONS
}

\author{
BY
}

AHMED I. ZAYED

\begin{abstract}
The results of Gilbert on the location of the singular points of an analytic function $f(z)$ given by Gegenbauer (ultraspherical) series expansion $f(z)=$ $\sum_{n=0}^{\infty} a_{n} C_{n}^{\mu}(z)$ are extended to the case where the series converges to a distribution. On the other hand, this generalizes Walter's results on distributions given by Legendre series: $f(z)=\sum_{n=0}^{\infty} a_{n} C_{n}^{1 / 2}(z)$. The singularities of the analytic representation of $f(z)$ are compared to those of the associated power series $g(z)=$ $\sum_{n=0}^{\infty} a_{n} z^{n}$. The notion of value of a distribution at a point is used to study the boundary behavior of the associated power series. A sufficient condition for Abel summability of Gegenbauer series is also obtained in terms of the distribution to which the series converges.
\end{abstract}

1. Introduction. Since the publication of the fundamental paper by Nehari [11] on the location of the singular points of an analytic function given by a Legendre series, a certain amount of work has been devoted by various people to finding the singularities of analytic functions given by eigenfunction series expansions. Nehari's proof used the same kind of argument that Hadamard [9] originally used in his proof of the "multiplication of singularities" theorem. This argument, which is usually referred to as Hadamard's argument, has been exploited extensively by Gilbert [3], [4] (also see these references for bibliography), and by Gilbert and Howard [7], [8] in a series of papers studying the analytic properties of solutions of some partial differential equations. In studying the singularities of an analytic function $f(z)$ given by the eigenfunction series expansion $f(z)=\sum_{n=0}^{\infty} a_{n} v_{n}(z)$, they used Nehari's technique to get information about the locations of the singular points of $f(z)$ by comparing them to the locations of the singular points of the associated power series $\phi(z)=\sum_{n=0}^{\infty} a_{n} z^{n}$. The cases where the eigenfunctions $\left\{v_{n}(z)\right\}_{n=0}^{\infty}$ are Gegenbauer (ultraspherical) polynomials, Jacobi polynomials, and eigenfunctions of certain Sturm-Liouville systems have been investigated in [5], [6] and $[8]$ respectively.

In all the previously-mentioned cases the proof depends on the fact that the series of eigenfunctions converges to a function $f(z)$ analytic in a complex neighborhood of a real interval. However, if the function has a singular point in this interval, the proof fails. G. Walter [13] was the first to attack the problem of

Received by the editors August 27, 1979.

AMS (MOS) subject classifications (1970). Primary 46F12; Secondary 33A50.

Key words and phrases. Gegenbauer expansions, generalized functions, value of a distribution at a point, Abel summability. $0002-9947 / 80 / 0000-0560 / \$ 05.25$ 
generalizing these results to the case where the eigenfunction expansion $f(z)=$ $\sum_{n=0}^{\infty} a_{n} v_{n}(z)$ has singular points in the real interval. In fact, he considered the case where $\left\{v_{n}(z)\right\}_{n=0}^{\infty}$ are Legendre polynomials. He considered not only the case where $f(z)$ has real singularities but also the case where $f(z)$ is not even a function, but rather a generalized function (Schwartz distribution). Evidently, in the case where the eigenfunction expansion does not converge to a function, the singularities of $f(z)$ must be interpreted differently. It turns out that in some cases it is possible to find an analytic function $\hat{f}(z)$ whose singularities can be related to those of the associated power series in a fashion similar to that obtained by the authors cited previously. In $\$ 2$ of this paper, which deals mainly with eigenfunction expansions of the form $\sum_{n=0}^{\infty} a_{n} C_{n}^{\mu}(x)$ where $C_{n}^{\mu}(x)$ is the Gegenbauer (ultraspherical) polynomial of degree $n$, we show that this is indeed possible for these expansions. This is done by using the integral operator method together with Hadamard's argument. The results of this section are on one hand an extension of Gilbert's results [5], and a generalization of Walter's on the other.

In $\$ 3$ we use different techniques to get more information about the relationship between the analytic properties of the power series and those of the Gegenbauer series. For example, having characterized the singular points of the power series, one may ask what about the regular points?: Does $\phi(z)$ approach its limit $\phi(\beta)$ as $z$ approaches $\beta$ radially, where $\beta$ is a point on the boundary of the disk of convergence? The answer will be shown to be affirmative provided that the Gegenbauer series behaves nicely at the point $\frac{1}{2}(\beta+1 / \beta)$. The technique that will be used is the technique of value of a distribution at a point. We shall also employ this technique to derive a sufficient condition for the Abel summability of Gegenbauer series.

\section{Singularities of ultraspherical expansions.}

2.0. Preliminaries and notations. We begin by recalling some of the basic properties of the ultraspherical polynomials $C_{n}^{\mu}(x)$. Fix $\mu>0$, then $C_{n}^{\mu}(x), n=$ $0,1,2, \ldots$, are defined by the generating relation

$$
\sum_{n=0}^{\infty} C_{n}^{\mu}(x) Z^{n}=\left(1-2 x Z+Z^{2}\right)^{-\mu}, \quad|Z|<1, x \in[-1,1] .
$$

The set $\left\{C_{n}^{\mu}(x)\right\}_{n=0}^{\infty}$ is orthogonal and complete over $(-1,1)$ with respect to the measure $\left(1-x^{2}\right)^{\mu-1 / 2} d x$. In fact, we have

$$
\int_{-1}^{1} C_{n}^{\mu}(x) C_{m}^{\mu}(x)\left(1-x^{2}\right)^{\mu-1 / 2} d x=h_{n}^{\mu} \delta_{n, m}
$$

where

$$
h_{n}^{\mu}=\frac{2^{1-2 \mu} \pi \Gamma(n+2 \mu)}{n !(\mu+n)[\Gamma(\mu)]^{2}} .
$$

It is also known that $C_{n}^{\mu}(x)$ satisfies the differential equation

$$
\left(1-x^{2}\right) y^{\prime \prime}-(2 \mu+1) x y^{\prime}+n(n+2 \mu) y=0 .
$$


We denote by $L$ the differential operator

$$
L=\left(1-x^{2}\right) d^{2} / d x^{2}-(2 \mu+1) x d / d x-\mu^{2},
$$

which we call the Gegenbauer differential operator. Evidently,

$$
L C_{n}^{\mu}(x)=-(n+\mu)^{2} C_{n}^{\mu}(x)
$$

The following two relations are also useful and will be used later.

$$
\begin{aligned}
\sum_{n=0}^{\infty}(n+\mu) C_{n}^{\mu}(x) r^{n} & =\frac{\mu\left(1-r^{2}\right)}{\left(1-2 x r+r^{2}\right)^{\mu+1}}, \quad|r|<1, \\
\frac{\Gamma^{2}(\mu) 2^{2 \mu-1} n !}{\Gamma(n+2 \mu)} C_{n}^{\mu}(x) C_{n}^{\mu}(y) & =\int_{-1}^{1}\left(1-t^{2}\right)^{\mu-1} C_{n}^{\mu}(\cos W) d t
\end{aligned}
$$

where $\cos W=x y+t \sqrt{\left(1-x^{2}\right)\left(1-y^{2}\right)}, \operatorname{Re} \mu>0$.

For more details on the ultraspherical polynomials see [2] and [12].

2.1. Singularity theorem. Borrowing the original Hadamard idea, Nehari [11] proved the following theorem: Let $\left\{a_{n}\right\}$ be a sequence of complex numbers such that

$$
\varlimsup_{n \rightarrow \infty}\left|a_{n}\right|^{1 / n}=\rho<1
$$

and let $f(t), \phi(z)$ be defined by the expansions

$$
f(t)=\sum_{n=0}^{\infty} a_{n} P_{n}(t), \quad|t+1|+|t-1|<\frac{1+\rho^{2}}{\rho},
$$

$\left(P_{n}(t)\right.$ is Legendre polynomial of degree $\left.n\right)$

$$
\phi(z)=\sum_{n=0}^{\infty} a_{n} z^{n}, \quad|z|<\rho^{-1}
$$

which converge in the regions indicated, then a point $\tau \neq \pm 1$ is a singular point of $f(t)$ if and only if $\tau=\frac{1}{2}(\sigma+1 / \sigma)$ where $\sigma$ is a singular point of $\phi(z)$.

The proof of Nehari's theorem depends essentially on the fact that $\overline{\lim }_{n \rightarrow \infty}\left|a_{n}\right|^{1 / n}$ $=\rho<1$. But if $\varlimsup_{n \rightarrow \infty}\left|a_{n}\right|^{1 / n}=\rho=1$ the proof fails.

In fact this is not surprising at all since the series $\sum_{n=0}^{\infty} a_{n} P_{n}(t)$ may diverge everywhere. The case where $\varlimsup_{n \rightarrow \infty}\left|a_{n}\right|^{1 / n}=1$ with $a_{n}=O\left(n^{P}\right)$ for some integer $P$ was investigated by Walter [13]. He showed that the series $\sum_{n=0}^{\infty} a_{n} P_{n}(t)$ converges in $(-1,1)$ to a distribution $f$ whose analytic representation $\hat{f}(\xi)$ is given by the series $\hat{f}(\xi)=\sum_{n=0}^{\infty}(i / \pi) a_{n} Q_{n}(\xi)$ for $\operatorname{Im} \xi \neq 0$, where $Q_{n}(\xi)$ is the Legendre function of the second kind. In addition to that, $\hat{f}(\xi)$ has a singular point at $\xi=\frac{1}{2}(\sigma+1 / \sigma)$ in $(-1,1)$ if and only if $\phi(z)=\sum_{n=0}^{\infty} a_{n} z^{n}$ has a singular point on the unit circle at $\sigma \neq \pm 1$ and at $\bar{\sigma}$.

Analogous to, but more general than, Nehari's result is the following theorem due to Gilbert [5]: Let $\left\{a_{n}\right\}_{n=0}^{\infty}$ be a sequence of complex numbers such that $\varlimsup_{n \rightarrow \infty}\left|a_{n}\right|^{1 / n}=\rho<1$, and let $f(t)$ and $\phi(z)$ be given by

$$
f(t)=\sum_{n=0}^{\infty} a_{n} C_{n}^{\mu}(t), \quad|t-1|+|t+1|<\frac{1+\rho^{2}}{\rho},
$$


where $C_{n}^{\mu}(t)$ is Gegenbauer polynomial of degree $n$, and

$$
\phi(z)=\sum_{n=0}^{\infty} \frac{\Gamma(n+2 \mu)}{\Gamma(2 \mu) n !} a_{n} z^{n}, \quad|z|<\rho^{-1} .
$$

Then $f(t)$ has a singular point at $t=\tau, \tau \neq \pm 1$ if and only if $\tau=\frac{1}{2}(\sigma+1 / \sigma)$ where $\sigma$ is a singular point of $\phi(z)$.

We obtain Nehari's theorem as a special case of Gilbert's by setting $\mu=\frac{1}{2}$ since $C_{n}^{1 / 2}(x)=P_{n}(x)$. The main purpose of this section is to extend Gilbert's theorem to the case where $\overline{\lim }_{n \rightarrow \infty}\left|a_{n}\right|^{1 / n}=1$ and $a_{n}=O\left(n^{p}\right)$ for some integer $P$. From this generalization, which is done in a fashion similar to the one given by Walter for Legendre series, we can obtain Walter's result as a special case by setting $\mu=\frac{1}{2}$. Indeed some difficulties arise in the extension due to the fact that the Gegenbauer operator $L$ is not selfadjoint. In fact, $L$ is selfadjoint if and only if $\mu=\frac{1}{2}$, i.e. $C_{n}^{1 / 2}(t)=P_{n}(t)$

One side effect of this is the lack of symmetry in some of the results which was found to a greater degree in Walter's results.

THEOREM (2.1). Let $\left\{a_{n}\right\}_{n=0}^{\infty}$ be a sequence of complex numbers such that

$$
\left|a_{n}\right| \leqslant M(n+1)^{P}, \quad n=0,1,2,3, \ldots,
$$

for some integers $M$ and $P$. Then there exists a distribution $f$ with support in $[-1,1]$ such that:

(i) The series $\sum_{n=0}^{\infty} a_{n} C_{n}^{\mu}(t)$ converges in $(-1,1)$ to $f$.

(ii) If either $f$ has a compact support in $(-1,1)$ or $\mu-\frac{1}{2}$ is an integer, then $g(t)=\left(1-t^{2}\right)^{\mu-1 / 2} f(t)$ is also a distribution with support in $[-1,1]$.

(iii) The analytic representation $\hat{g}(\xi)$ of $g$ is given by the series $\hat{g}(\xi)=$ $\sum_{n=0}^{\infty} a_{n} Q_{n}^{\mu}(\xi)$, where $\left(1-\xi^{2}\right)^{\mu-1 / 2} Q_{n}^{\mu}(\xi)$ are the Gegenbauer functions of the second kind, and $\hat{g}(\xi)$ is holomorphic in the $\xi$-plane cut along $[-1,1]$.

(iv) $\hat{g}(\xi)$ has a singular point at $\beta=\frac{1}{2}(\alpha+1 / \alpha)$ in $(-1,1)$ if and only if

$$
\phi(z)=\sum_{n=0}^{\infty} \frac{\Gamma(n+2 \mu)}{n !} a_{n} z^{n}
$$

has one at $z=\alpha$ on the unit circle and $\alpha \neq \pm 1$.

Proof. (i) Consider the function

$$
F(t)=\sum_{n=0}^{\infty} \frac{(-1)^{k} a_{n}}{(n+\mu)^{2 k}} C_{n}^{\mu}(t), \quad t \in[-1,1],
$$

and zero otherwise, where $2 k \geqslant 2 \mu+P+1 . F(t)$ is continuous on $[-1,1]$ since $\left|C_{n}^{\mu}(t)\right| \leqslant A n^{2 \mu-1}$ and hence we have

$$
|F(t)| \leqslant A \sum_{n=0}^{\infty} \frac{M(n+1)^{P}}{(n+\mu)^{2 k}} n^{2 \mu-1} \leqslant A \sum_{n=0}^{\infty} \frac{2^{P} M n^{2 \mu+P-1}}{(n+\mu)^{2 k}} .
$$

The last series converges by our choice of $k$. Since the Gegenbauer differential operator

$$
L=\left(1-t^{2}\right) d^{2} / d t^{2}-(2 \mu+1) t d / d t-\mu^{2}
$$


is continuous in $\mathscr{Q}^{*}$ (the space of distributions) and the defining series of $F(t)$ converges uniformly, hence, converges in $\mathscr{D}^{*}$, then it follows that

$$
f=L^{k} F(t)=\sum_{n=0}^{\infty} a_{n} C_{n}^{\mu}(t) \quad \text { in }(-1,1) .
$$

Clearly, supp $f \subseteq[-1,1]$.

(ii) If $\mu-\frac{1}{2}$ is an integer, then $\left(1-t^{2}\right)^{\mu-1 / 2}$ is a $C^{\infty}$-function, hence a multiplier of $f$. If $\operatorname{supp} f \subset(-1,1)$, then $\left(1-t^{2}\right)^{\mu-1 / 2} f$ is also a $C^{\infty}$-function in a neighborhood of supp $f$, for all $\mu$.

(iii) Since $g$ has compact support, then the analytic representation $\hat{g}(\xi)$ of $g$ is given by $\hat{g}(\xi)=(1 / 2 \pi i)(g, 1 /(t-\xi)), \xi \notin \operatorname{supp} g$ (see Bremermann [1]). Since $1 /(t-\xi)$ is analytic in $[-1,1]$ for $\operatorname{Im} \xi \neq 0$, then its expansion in terms of Gegenbauer polynomials converges uniformly to it. In fact, since $1 /(t-\xi)$ is analytic in some ellipse $E_{\xi}$ with foci at \pm 1 , then its expansion in a Gegenbauer series and all its derivatives converge uniformly on compact subsets of $E_{\xi}$ [12]. Hence, the convergence of the series

$$
\frac{\lambda(t)}{t-\xi}=\sum_{n=0}^{\infty}\left(\frac{1}{h_{n}^{\mu}} \int_{-1}^{1} \frac{\left(1-u^{2}\right)^{\mu-1 / 2} C_{n}^{\mu}(u) d u}{u-\xi}\right) C_{n}^{\mu}(t) \lambda(t)
$$

where $\lambda(t) \in C_{0}^{\infty}, \lambda=1$ in $[-1,1]$ and supp $\lambda$ is in $E_{\xi} \cap R^{1}$, may be regarded as convergence in the sense of $\mathscr{D}$. Therefore, we have

$$
\begin{aligned}
\hat{g}(\xi) & =(1 / 2 \pi i)(g, \lambda(t) /(t-\xi))=(1 / 2 \pi i)(g, 1 /(t-\xi)) \\
& =\frac{1}{2 \pi i}\left(g, \sum_{n=0}^{\infty}\left(\frac{1}{h_{n}^{\mu}} \int_{-1}^{1} \frac{\left(1-u^{2}\right)^{\mu-1 / 2}}{(u-\xi)} C_{n}^{\mu}(u) d u\right) C_{n}^{\mu}(t)\right) \\
& =\frac{1}{2 \pi i} \sum_{n=0}^{\infty} \frac{1}{h_{n}^{\mu}} Q_{n}^{\mu}(\xi)\left(g, C_{n}^{\mu}(t)\right), \quad \xi \notin[-1,1],
\end{aligned}
$$

where

$$
Q_{n}^{\mu}(\xi)=\int_{-1}^{1} \frac{\left(1-u^{2}\right)^{\mu-1 / 2}}{(u-\xi)} C_{n}^{\mu}(u) d u, \quad \mu>-\frac{1}{2}, \mu \neq 0,
$$

and $\left(1-\xi^{2}\right)^{\mu-1 / 2} Q_{n}^{\mu}(\xi)$ is known as the Gegenbauer function of the second kind (see [12]). But

$$
\begin{aligned}
\left(g, C_{n}^{\mu}(t)\right) & =\left(\left(1-t^{2}\right)^{\mu-1 / 2} f(t), C_{n}^{\mu}(t)\right)=\left(f,\left(1-t^{2}\right)^{\mu-1 / 2} C_{n}^{\mu}(t)\right) \\
& =\left(L^{k} F,\left(1-t^{2}\right)^{\mu-1 / 2} C_{n}^{\mu}(t)\right)=\left(F, L^{* k}\left(1-t^{2}\right)^{\mu-1 / 2} C_{n}^{\mu}(t)\right) .
\end{aligned}
$$

On the other hand, it is not hard to see that the adjoint operator $L^{*}$ of $L$ is given by

$$
L^{*}=\left(1-t^{2}\right) d^{2} / d t^{2}+(2 \mu-3) t d / d t-(\mu-1)^{2}
$$

and that

$$
L^{*}\left(1-t^{2}\right)^{\mu-1 / 2} C_{n}^{\mu}(t)=-(n+\mu)^{2}\left(1-t^{2}\right)^{\mu-1 / 2} C_{n}^{\mu}(t)
$$


Thus, by substituting equation (2.15) in (2.13) and using (2.9) one gets

$$
\begin{aligned}
\left(g, C_{n}^{\mu}(t)\right) & =(-1)^{k}(n+\mu)^{2 k}\left(F,\left(1-t^{2}\right)^{\mu-1 / 2} C_{n}^{\mu}(t)\right) \\
& =(-1)^{k}(n+\mu)^{2 k}\left((-1)^{k} /(n+\mu)^{2 k}\right) a_{n} h_{n}^{\mu}=a_{n} h_{n}^{\mu},
\end{aligned}
$$

and consequently

$$
\hat{g}(\xi)=\frac{1}{2 \pi i} \sum_{n=0}^{\infty} a_{n} Q_{n}^{\mu}(\xi), \quad \xi \notin[-1,1] .
$$

To show that $\hat{g}(\xi)$ is analytic in the cut plane, we need to estimate $Q_{n}^{\mu}(\xi)$ as follows:

$$
\begin{aligned}
\left|Q_{n}^{\mu}(\xi)\right| & =\left|\int_{-1}^{1} \frac{\left(1-t^{2}\right)^{\mu-1 / 2} C_{n}^{\mu}(t)}{(t-\xi)} d t\right| \\
& =\frac{1}{(n+\mu)^{2 k}}\left|\int_{-1}^{1} \frac{1}{t-\xi} L^{* k}\left(1-t^{2}\right)^{\mu-1 / 2} C_{n}^{\mu}(t) d t\right| \\
& =\frac{1}{(n+\mu)^{2 k}}\left|\int_{-1}^{1}\left(1-t^{2}\right)^{\mu-1 / 2} C_{n}^{\mu}(t) L^{k} \frac{1}{(t-\xi)} d t\right|<\frac{n^{2 \mu-1} M(\xi)}{(n+\mu)^{2 k}},
\end{aligned}
$$

where $M(\xi)$ is a constant that depends on $\xi$ but not $n$. Therefore,

$$
\left|\sum_{n=0}^{\infty} a_{n} Q_{n}^{\mu}(\xi)\right| \leqslant \sum_{n=0}^{\infty}\left|a_{n}\right|\left|Q_{n}^{\mu}(\xi)\right| \leqslant \sum_{n=0}^{\infty} \frac{M(n+1)^{P} n^{2 \mu-1} M(\xi)}{(n+\mu)^{2 k}}<\infty,
$$

and hence the series converges absolutely and by standard argument it can be shown that it converges uniformly on compact subsets of the cut plane.

(iv) First we show that the function

$$
K(z, \xi)=\sum_{n=0}^{\infty} Q_{n}^{\mu}(\xi) z^{-n}
$$

which is analytic for $|z|>1$ and $\xi \notin[-1,1]$ can be continued analytically so that the only possible singularities in the set $\{(z, \xi) \mid(z, \xi) \in \mathbf{C} \times \mathbf{C}$ and $|z|>1\}$ are at $\xi= \pm 1$ and $\xi=\frac{1}{2}(z+1 / z)$. The argument used in the proof is similar to the one given by Walter. It goes like this.

$$
\begin{aligned}
K(z, \xi) & =\sum_{n=0}^{\infty} z^{-n} \int_{-1}^{1} \frac{\left(1-t^{2}\right)^{\mu-1 / 2}}{(t-\xi)} C_{n}^{\mu}(t) d t \\
& =\int_{-1}^{1} \frac{\left(1-t^{2}\right)^{\mu-1 / 2}}{(t-\xi)} \sum_{n=0}^{\infty} z^{-n} C_{n}^{\mu}(t) d t \\
& =\int_{-1}^{1} \frac{\left(1-t^{2}\right)^{\mu-1 / 2} z^{2 \mu}}{(t-\xi)\left(1-2 t z+z^{2}\right)^{\mu}} d t
\end{aligned}
$$

Interchanging the summation and integration is possible since the series $\sum_{n=0}^{\infty} z^{-n} C_{n}^{\mu}(t)$ converges uniformly to $z^{2 \mu} /\left(1-2 t z+z^{2}\right)^{\mu}$ for $t \in[-1,1]$ and fixed $|z|>1$. Now by using the Hadamard argument we deduce that $K(z, \xi)$ has possible singular points only at $\xi= \pm 1$ and at the common singular points of $1 /(t-\xi)$ and $1 /\left(1-2 t z+z^{2}\right)^{\mu}$ i.e., $\xi=\frac{1}{2}(z+1 / z)$.

Now we use $K(z, \xi)$ to construct an integral operator that maps $\hat{g}(\xi)$ into 
$\tilde{\phi}(z)=\sum_{n=0}^{\infty} a_{n} z^{n}$. Consider

$$
\hat{g}(\xi)=\frac{1}{2 \pi i} \sum_{n=0}^{\infty} a_{n} Q_{n}^{\mu}(\xi)=\frac{1}{(2 \pi i)^{2}} \sum_{n=0}^{\infty} Q_{n}^{\mu}(\xi) \sum_{k=0}^{\infty} a_{k} \int_{\gamma} z^{k-n} \frac{d z}{z}
$$

where $\gamma$ is the contour $|z|=\rho<1$ and the last expansion is obtained from the observation that

$$
a_{n}=\frac{1}{2 \pi i} \sum_{k=0}^{\infty} a_{k} \int_{\gamma} z^{k-n} \frac{d z}{z}
$$

Since the series $\tilde{\phi}(z)=\sum_{k=0}^{\infty} a_{k} z^{k}$ converges uniformly on $\gamma$ and because $\left|z^{-1}\right|>1$ we can interchange the sums and the integral to obtain

$$
\hat{g}(\xi)=\frac{-1}{4 \pi^{2}} \int_{\gamma}\left(\sum_{n=0}^{\infty} Q_{n}^{\mu}(\xi) z^{-n}\right)\left(\sum_{k=0}^{\infty} a_{k} z^{k}\right) \frac{d z}{z}=\frac{-1}{4 \pi^{2}} \int_{\gamma} K(z, \xi) \tilde{\phi}(z) \frac{d z}{z} .
$$

This is valid for $\operatorname{Im} \xi$ sufficiently large since $K(z, \xi)=\sum_{n=0}^{\infty} Q_{n}^{\mu}(\xi) z^{-n}$ converges uniformly in $|z|=\rho<1$ for $\operatorname{Im} \xi$ sufficiently large by the asymptotic formula $Q_{n}^{\mu}(\xi)=O\left(\xi^{-n}\right)[12]$.

Again by the Hadamard argument we can show that $\hat{g}(\xi)$ is analytic for all $\xi$ except possibly at $\xi= \pm 1$ and the common singularities of $K(z, \xi)$ and $\tilde{\phi}(z)$. Thus, if $\tilde{\phi}(z)$ has a singular point at $z=\alpha$, then $\hat{g}(\xi)$ may have one only at $\beta=$ $\frac{1}{2}(\alpha+1 / \alpha)$ except at \pm 1 .

On the other hand, if $\phi(z)$ has a singular point at $z=\alpha$, then so does $\tilde{\phi}(z)$ and conversely. This follows from the original Hadamard's theorem applied to the series

$$
\sum_{n=0}^{\infty} \frac{\Gamma(n+2 \mu)}{n !} a_{n} z^{n}, \quad \sum_{n=0}^{\infty} \frac{\Gamma(n+2 \mu)}{n !} z^{n}, \quad \sum_{n=0} a_{n} z^{n} .
$$

Conversely, since $g$ has compact support, then by the representation theorem for distributions [1], we have

$$
\lim _{\varepsilon \rightarrow 0} \int[\hat{g}(t+i \varepsilon)-\hat{g}(t-i \varepsilon)] \psi(t) d t=(g, \psi) \quad \text { for all } \psi \in C^{\infty} \text {. }
$$

Choosing $\psi(t)=A\left(1-z^{2}\right) /\left(1-2 t z+z^{2}\right)^{\mu+1}$, where $A=\mu \Gamma^{2}(\mu) / \pi 2^{1-2 \mu}$ and $|z|$ $<1$, we get

$$
\begin{aligned}
\left(g(t), \frac{A\left(1-z^{2}\right)}{\left(1-2 t z+z^{2}\right)^{\mu+1}}\right) & =\left(\left(1-t^{2}\right)^{\mu-1 / 2} f(t), \frac{A\left(1-z^{2}\right)}{\left(1-2 t z+z^{2}\right)^{\mu+1}}\right) \\
& =\left(\sum_{n=0}^{\infty} a_{n} C_{n}^{\mu}(t), \frac{A\left(1-t^{2}\right)^{\mu-1 / 2}\left(1-z^{2}\right)}{\left(1-2 t z+z^{2}\right)^{\mu+1}}\right) \\
& =\left(\sum_{n=0}^{\infty} a_{n} C_{n}^{\mu}(t), \frac{A}{\mu}\left(1-t^{2}\right)^{\mu-1 / 2} \sum_{k=0}^{\infty}(k+\mu) C_{k}^{\mu}(t) z^{k}\right) \\
& =\sum_{n=0}^{\infty} a_{n} \frac{A}{\mu} \sum_{k=0}^{\infty}(k+\mu) z^{k} h_{k}^{\mu} \delta_{k, n} \\
& =\sum_{n=0}^{\infty} \frac{\Gamma(n+2 \mu)}{n !} a_{n} z^{n}=\phi(z) .
\end{aligned}
$$


Let

$$
\phi_{\varepsilon}(z)=\int_{-1}^{1} \frac{\left(1-z^{2}\right)}{\left(1-2 z t+z^{2}\right)^{\mu+1}}[\hat{g}(t+i \varepsilon)-\hat{g}(t-i \varepsilon)] d t
$$

This integral can be expressed as a contour integral with end points at -1 and 1 so that the contour avoids the singularities of the integrand. We invoke the Hadamard argument once more to conclude that $\phi_{\varepsilon}(z)$ is analytic except possibly at a zero of $1-2(\beta \pm i \varepsilon) z+z^{2}$, where $\beta$ is a singular point of $\hat{g}(\xi)$. Taking the limit as $\varepsilon \rightarrow 0$, we deduce that $\phi(z)$ is analytic except possibly at $\alpha$ where $\beta=\frac{1}{2}(\alpha+1 / \alpha)$. Q.E.D.

\section{On the Abel summability of Gegenbauer series: Point values of a distribution. In} this section we use the concept of value of a distribution at a point as introduced by Lojasiewicz [10].

Definition. Let $f$ be a distribution on $R$. Then $f$ has a value at $x_{0}$ if and only if $\lim _{\lambda \rightarrow 0} f\left(\lambda x+x_{0}\right)$ exists in the sense of distributions. A point at which the distribution has a value is said to be regular, otherwise it is singular.

It has been proved that a distribution $f(x)$ has the value $\gamma$ at a point $x_{0}$ if and only if there exist an integer $k \geqslant 0$ and a continuous function $F(x)$ such that $F^{(k)}=f$ and $\lim _{x \rightarrow x_{0}} F(x) /\left(x-x_{0}\right)^{k}=\gamma / k !$.

In this section we utilize the concept of value of a distribution at a point to extend the results of the last section. In Theorem (3.2) we look at the boundary behavior of the power series $\phi(z)=\sum_{n=0}^{\infty} a_{n} h_{n}^{\mu} z^{n}$ as $z \rightarrow \beta$ radially; $|\beta|=1$, as it is related to the behavior of the associated series of ultraspherical polynomials $f=\sum_{n=0}^{\infty} a_{n} C_{n}^{\mu}(t)$. More specifically, we will show that if $f^{([\mu \mathrm{D})}$ which is a distribution in general has a value $\gamma$ at the point $\alpha \in(-1,1)$ then $\phi(z) \rightarrow \phi(\beta)$ as $z \rightarrow \beta$ radially where $\beta$ is the image of $\alpha$ under the conformal mapping

$$
z=t+\left(t^{2}-1\right)^{1 / 2}
$$

We also investigate the Abel summability of series of ultraspherical polynomials. It will be shown that the series $\sum_{n=0}^{\infty} a_{n} C_{n}^{\mu}(\alpha), \alpha \in(-1,1)$, is Abel summable to $\gamma$ whenever the distribution $\sum_{n=0}^{\infty} a_{n} C_{n}^{\mu}(x)$ has a value $\gamma$ at $\alpha$. We shall use the following two lemmas which are well known.

Lemma (3.1). Let $f \in L^{1}[-1,1], \lim _{t \rightarrow \alpha} f(t)=\gamma$ where $\alpha \in(-1,1)$ and suppose that there exists a summability kernel $K(\alpha, t, r)$ with the following properties.

(i) $K(\alpha, t, r) \rightarrow 0$ uniformly for $|t-\alpha| \geqslant \varepsilon>0$ as $r \rightarrow 1^{-}$.

(ii) $\int_{-1}^{1}|K(\alpha, t, r)| d t \leqslant M_{\alpha}$ a constant for $r \in(0,1)$ and $\alpha \in(-1,1)$.

(iii) $\int_{a}^{b} K(\alpha, t, r) d t \rightarrow 1$ as $r \rightarrow 1^{-} ; \alpha \in(a, b)$ and $[a, b] \subset(-1,1)$. Then $\int_{-1}^{1} f(t) K(\alpha, t, r) d t \rightarrow \gamma$ as $r \rightarrow 1^{-}$.

Proof. See Zygmund [15].

A summability kernel $K(x, t, r)$ that satisfies the three conditions of Lemma (3.1) is called quasi-positive. 
Lemma (3.2). Let $f \in L^{1}(-1,1)$; then the Abel sum $f(x, r)$ of the series

$$
f(x) \sim \sum_{n=0}^{\infty} a_{n} C_{n}^{\mu}(x) \text { where } a_{n}=\frac{1}{h_{n}^{\mu}} \int_{-1}^{1}\left(1-t^{2}\right)^{\mu-1 / 2} f(t) C_{n}^{\mu}(t) d t
$$

is given by

$$
f(r, x)=\frac{\mu}{\pi} \int_{-1}^{1} \int_{-1}^{1}\left(1-t^{2}\right)^{\mu-1 / 2}\left(1-u^{2}\right)^{\mu-1} \frac{\left(1-r^{2}\right)}{\left(1-2 r \cos \gamma+r^{2}\right)^{\mu+1}} f(t) d t d u
$$

where $\cos \gamma=x t+u \sqrt{\left(1-t^{2}\right)\left(1-x^{2}\right)}, 0 \leqslant r<1$.

SKETCH OF THE PROOF.

$$
\begin{aligned}
f(x, r) & =\sum_{n=0}^{\infty} a_{n} C_{n}^{\mu}(x) r^{r}=\sum_{n=0}^{\infty} \frac{1}{h_{n}^{\mu}} C_{n}^{\mu}(x) r^{n} \int_{-1}^{1}\left(1-t^{2}\right)^{\mu-1 / 2} f(t) C_{n}^{\mu}(t) d t \\
& =\sum_{n=0}^{\infty} \frac{1}{h_{n}^{\mu}} r^{n} \int_{-1}^{1} f(t)\left(1-t^{2}\right)^{\mu-1 / 2} C_{n}^{\mu}(x) C_{n}^{\mu}(t) d t
\end{aligned}
$$

On account of relations (2.7), (2.8) and (3.1) the result follows easily.

Let us denote the kernel of the Abel summability by $H(x, t, r)$ i.e.,

$$
\begin{aligned}
H(x, t, r) & =\sum_{n=0}^{\infty} \frac{1}{h_{n}^{\mu}}\left(1-t^{2}\right)^{\mu-1 / 2} C_{n}^{\mu}(x) C_{n}^{\mu}(t) r^{n} \\
& =\frac{\mu}{\pi} \int_{-1}^{1}\left(1-t^{2}\right)^{\mu-1 / 2}\left(1-u^{2}\right)^{\mu-1} \frac{\left(1-r^{2}\right)}{\left(1-2 r \cos \gamma+r^{2}\right)^{\mu+1}} d u
\end{aligned}
$$

and let us also define

$$
\begin{gathered}
G^{P}(x, t, r)=(-1)^{p} \frac{(x-t)^{P}}{P !} \sum_{n=0}^{\infty} \frac{1}{h_{n}^{\mu}} C_{n}^{\mu}(x) \frac{d^{P}}{d t^{P}}\left(1-t^{2}\right)^{\mu-1 / 2} C_{n}^{\mu}(t) r^{n} \\
=\frac{(-1)^{P} \mu}{\pi} \frac{(x-t)^{P}}{P !} \int_{-1}^{1}\left(1-r^{2}\right)\left(1-u^{2}\right)^{\mu-1} \frac{d^{P}}{d t^{P}} \frac{\left(1-t^{2}\right)^{\mu-1 / 2}}{\left(1-2 r \cos \gamma+r^{2}\right)^{\mu+1}} d u
\end{gathered}
$$

Some important properties of $G^{P}(x, t, r)$ are given in Lemma (3.4).

LEMMA (3.3).

$$
\text { (i) } \frac{d^{P}}{d t^{P}} \frac{\left(1-t^{2}\right)^{\mu-1 / 2}}{\left(1-2 r \cos \gamma+r^{2}\right)^{\mu+1}}=\frac{\left(1-t^{2}\right)^{\mu-1 / 2-P}}{\left(1-2 r \cos \gamma+r^{2}\right)^{\mu+1+P}} P(t, \gamma, r)
$$

where $P(t, \gamma, r)$ is a polynomial in $t$ and $r$ with coefficients as bounded functions of $\cos \gamma,(\partial \cos \gamma / \partial t), \ldots,(\partial \cos \gamma / \partial t)^{P},\left(\partial^{2} \cos \gamma / \partial t^{2}\right), \ldots,\left(\partial^{P} \cos \gamma / \partial t^{P}\right)$. 
(ii) $\frac{d^{P}}{d t^{P}} \frac{\left(1-t^{2}\right)^{\mu-1 / 2}}{\left(1-2 r \cos \gamma+r^{2}\right)^{\mu+1}}$

$$
\begin{aligned}
= & \sum_{k=0}^{k<[P / 2]} \frac{a_{k}(t, \gamma, r)\left(1-t^{2}\right)^{\mu-1 / 2-k}}{\left(1-2 r \cos \gamma+r^{2}\right)^{\mu+1+P-k}}\left(\frac{\partial \cos \gamma}{\partial t}\right)^{P-2 k} \\
& +\frac{\left(1-t^{2}\right)^{\mu-1 / 2-P} A(t, \gamma, r)}{\left(1-2 r \cos \gamma+r^{2}\right)^{\mu+1+\eta}}
\end{aligned}
$$

where $A(t, \gamma, r)$ and $a_{k}(t, \gamma, r)$ are of the same type as $P(t, \gamma, r)$ in part (i) and $\eta=(P-2) / 2$ or $(P-1) / 2$ according to whether $P$ is even or odd.

PROof. For simplicity we write $R=\left(1-2 r \cos \gamma+r^{2}\right), u=\left(1-t^{2}\right), \nu=\mu+$ $1, \lambda=\mu-\frac{1}{2}$. We prove the lemma by induction on $P$.

(i) The formula is true for $P=1$ since

$$
\begin{aligned}
& \frac{d}{d t} \frac{\left(1-t^{2}\right)^{\mu-1 / 2}}{\left(1-2 r \cos \gamma+r^{2}\right)^{\mu+1}}=\frac{-(\mu+1)\left(1-t^{2}\right)^{\mu-1 / 2}(-2 r)}{\left(1-2 r \cos \gamma+r^{2}\right)^{\mu+2}}\left(\frac{\partial \cos \gamma}{\partial t}\right) \\
& \quad+\frac{\left(\mu-\frac{1}{2}\right)\left(1-t^{2}\right)^{\mu-3 / 2}(-2 t)}{\left(1-2 r \cos \gamma+r^{2}\right)^{\mu+1}} \\
& =\frac{\left(1-t^{2}\right)^{\mu-3 / 2} \backslash}{\left(1-2 r \cos \gamma+r^{2}\right)^{\mu+2}}\left[2 r(\mu+1)\left(1-t^{2}\right) \frac{\partial \cos \gamma}{\partial t}\right. \\
& \left.+\left(\mu-\frac{1}{2}\right)(-2 t)\left(1-2 r \cos \gamma+r^{2}\right)\right] .
\end{aligned}
$$

Assume it is true for $P$; then by taking the derivative of equation (3.6) one gets

$$
\begin{aligned}
\frac{d^{P+1}}{d t^{P+1}} \frac{u^{\lambda}}{R^{\nu}}= & \frac{d}{d t} \frac{u^{\lambda-P}}{R^{\nu+P}} P(t, \gamma, r)=\frac{-(\nu+P)(-2 r)(\partial \cos \gamma / \partial t) u^{\lambda-P}}{R^{\nu+P+1}} P(t, \gamma, r) \\
& +\frac{(\lambda-P)(-2 t) u^{\lambda-P-1}}{R^{\nu+P}} P(t, \gamma, r)+\frac{u^{\lambda-P}}{R^{\nu+P}} \frac{\partial P(t, \gamma, r)}{\partial t} \\
= & \frac{u^{\lambda-P-1}}{R^{\nu+P+1}}\left[(\nu+P)(2 r)\left(\frac{\partial \cos \gamma}{\partial t}\right) u P(t, \gamma, r)\right. \\
& \left.\quad+(\lambda-P)(-2 t) R P(t, \gamma, r)+u R \frac{\partial P}{\partial t}(t, \gamma, r)\right] \\
= & \frac{u^{\lambda-P-1}}{R^{\nu+P+1}} Q(t, \gamma, r),
\end{aligned}
$$

where $Q(t, \gamma, r)$ is a polynomial in $t$ and $r$ since $P(t, \gamma, r)$ is. Hence the formula is true for $P+1$. 
(ii) The formula is true for $P=1,2$, since

$$
\begin{aligned}
\frac{d}{d t} \frac{u^{\lambda}}{R^{\nu}}= & \frac{(-\nu)(-2 r) u^{\lambda}}{R^{\nu+1}}\left(\frac{\partial \cos \gamma}{\partial t}\right)+\frac{\lambda(-2 t) u^{\lambda-1}}{R^{\nu}} \\
\frac{d^{2}}{d t^{2}} \frac{u^{\lambda}}{R^{\nu}=} & \frac{(-2 r)^{2} \nu(\nu+1) u^{\lambda}}{R^{\nu+2}}\left(\frac{\partial \cos \gamma}{\partial t}\right)^{2}+\frac{(-\nu)(-2 r) \lambda(-2 t) u^{\lambda-1}}{R^{\nu+1}}\left(\frac{\partial \cos \gamma}{\partial t}\right) \\
& +\frac{(-\nu)(-2 r) u^{\lambda}}{R^{\nu+1}} \frac{\partial^{2} \cos \gamma}{\partial t^{2}}+\frac{(-\nu)(\lambda)(-2 t)(-2 r) u^{\lambda-1}}{R^{\nu+1}}\left(\frac{\partial \cos \gamma}{\partial t}\right) \\
& -\frac{2 \lambda u^{\lambda-1}}{R^{\nu}}+\frac{\lambda(\lambda-1)(-2 t)^{2} u^{\lambda-2}}{R^{\nu}} \\
= & \frac{a_{0}(t, r) u^{\lambda}}{R^{\nu+2}}\left(\frac{\partial \cos \gamma}{\partial t}\right)^{2}+\frac{a_{1}(t, r) u^{\lambda-1}}{R^{\nu+1}}+\frac{A(t, r) u^{\lambda-2}}{R^{\nu}}
\end{aligned}
$$

where $a_{0}, a_{1}$, and $A$ are polynomials satisfying the condition of the lemma. Assume it is true for $P$, then by taking the derivative of both sides of equation (3.7) one gets

$$
\begin{aligned}
& \frac{d^{P+1}}{d t^{P+1}} \frac{u^{\lambda}}{R^{\nu}}=\frac{d}{d t}\left(\sum_{k=0}^{k<[P / 2]} \frac{a_{k}(t, \gamma, r) u^{\lambda-k}}{R^{\nu+P-k}}\left(\frac{\partial \cos \gamma}{\partial t}\right)^{P-2 k}+\frac{u^{\lambda-P} A(t, \gamma, r)}{R^{\nu+\eta}}\right) \\
& =\sum_{k=0}^{k<[P / 2]} \frac{a_{k 1} u^{\lambda-k}}{R^{\nu+P-k+1}}\left(\frac{\partial \cos \gamma}{\partial t}\right)^{P-2 k+1} \\
& +\frac{a_{k 2} u^{\lambda-k-1}}{R^{\nu+P-k}}\left(\frac{\partial \cos \gamma}{\partial t}\right)^{P-2 k}+\frac{a_{k 3} u^{\lambda-k}}{R^{\nu+P-k}}\left(\frac{\partial \cos \gamma}{\partial t}\right)^{P-2 k-1} \\
& +\frac{a_{k 4} u^{\lambda-k}}{R^{\nu+P-k}}\left(\frac{\partial \cos \gamma}{\partial t}\right)^{P-2 k}+\frac{u^{\lambda-P} A_{1}}{R^{\nu+\eta+1}} \frac{\partial \cos \gamma}{\partial t}+\frac{u^{\lambda-P-1} A_{2}}{R^{\nu+\eta}} \\
& =\sum_{k=0}^{k<[P / 2]} \frac{a_{k 1} u^{\lambda-k}}{R^{\nu+(P+1)-k}}\left(\frac{\partial \cos \gamma}{\partial t}\right)^{(P+1)-2 k} \\
& +\frac{u^{\lambda-k-1}}{R^{\nu+P-k}}\left(\frac{\partial \cos \gamma}{\partial t}\right)^{P-2 k-1}\left(a_{k 2}\left(\frac{\partial \cos \gamma}{\partial t}\right)+a_{k 3} u+a_{k 4} u\left(\frac{\partial \cos \gamma}{\partial t}\right)\right) \\
& +\frac{u^{\lambda-P} A_{1}}{R^{\nu+\eta+1}} \frac{\partial \cos \gamma}{\partial t}+\frac{u^{\lambda-P-1}}{R^{\nu+\eta}} A_{2} \\
& =\sum_{k=0}^{k<[P / 2]} \frac{b_{k 1} u^{\lambda-k}}{R^{\nu+(P+1)-k}}\left(\frac{\partial \cos \gamma}{\partial t}\right)^{P+1-2 k} \\
& +\frac{b_{k 2} u^{\lambda-k-1}}{R^{\nu+P-k}}\left(\frac{\partial \cos \gamma}{\partial t}\right)^{P-2 k-1}+\frac{u^{\lambda-P} A_{1}}{R^{\nu+\eta+1}} \frac{\partial \cos \gamma}{\partial t}+\frac{u^{\lambda-P-1}}{R^{\nu+\eta}} A_{2}
\end{aligned}
$$

where $a_{k i}, i=1,2,3,4$, and $b_{k j}, A_{j}, j=1,2$, are all polynomials in $r$ and $t$ with coefficients as described in the statement of the lemma. Changing $k$ to $k-1$ in the second sum and combining similar terms, yield

$$
\frac{d^{P+1}}{d t^{P+1}} \frac{u^{\lambda}}{R^{\nu}}=\sum_{k=0}^{k<[P / 2]} \frac{B_{k} u^{\lambda-k}}{R^{\nu+(P+1)-k}}\left(\frac{\partial \cos \gamma}{\partial t}\right)^{(P+1)-2 k}+\frac{u^{\lambda-P-1}}{R^{\nu+\eta+1} B}
$$


where $B_{k}$ and $B$ are polynomials of the required type.

LEMMA (3.4). Let $x \in(-1,1)$; then $G^{P}(x, t, r)$ satisfies the following conditions.

(a) $G^{P}(x, t, r) \rightarrow 0$ uniformly for $0<\delta \leqslant \gamma \leqslant \pi$ as $r \rightarrow 1^{-}$; in particular, for $|t-x| \geqslant \delta_{1}>0$.

(b) $\int_{-1}^{1}\left|G^{P}(x, t, r)\right| d t \leqslant M_{x}$ a constant that depends only on $x$ for all $r \in(0,1)$.

(c) $\int_{a}^{b} G^{P}(x, t, r) d t \rightarrow 1$ as $r \rightarrow 1^{-}, x \in(a, b)$ and $[a, b] \subset(-1,1)$.

Proof. (a) From equations (3.5) and (3.6) we have

$$
\begin{aligned}
G^{P}(x, t, r)= & (-1)^{P} \frac{\mu}{\pi} \frac{(x-t)^{P}}{P !} \int_{-1}^{1}\left(1-r^{2}\right)\left(1-u^{2}\right)^{\mu-1} \\
& \cdot \frac{d^{P}}{d t^{P}} \frac{\left(1-t^{2}\right)^{\mu-1 / 2}}{\left(1-2 r \cos \gamma+r^{2}\right)^{\mu+1}} d u \\
= & (-1)^{P} \frac{\mu}{\pi} \frac{(x-t)^{P}}{P !} \int_{-1}^{1}\left(1-r^{2}\right)\left(1-u^{2}\right)^{\mu-1} \\
& \cdot \frac{\left(1-t^{2}\right)^{\mu-1 / 2-P}}{\left(1-2 r \cos \gamma+r^{2}\right)^{\mu+1+P} P(t, \gamma, r) d u} \\
= & (-1)^{P} \frac{\mu}{\pi} \frac{(x-t)^{P}}{P !} \int_{-1}^{1}\left(1-r^{2}\right)\left(1-u^{2}\right)^{\mu-1} \\
& \cdot \frac{\left(1-t^{2}\right)^{\mu-1 / 2-P}}{\left[(1-r)^{2}+4 r \sin ^{2}(\gamma / 2)\right]^{\mu+1+P}} P(t, \gamma, r) d u .
\end{aligned}
$$

Then, for $0<\delta \leqslant \gamma \leqslant \pi$, we have

$$
\begin{aligned}
\left|G^{P}(t, x, r)\right| \leqslant \frac{\mu}{\pi} & \frac{\left|(x-t)^{P}\right|}{P !} \frac{\left(1-r^{2}\right)\left(1-t^{2}\right)^{\mu-1 / 2-P}}{\left[(1-r)^{2}+4 r \sin ^{2}(\delta / 2)\right]^{\mu+1+P}} \\
& \cdot \int_{-1}^{1}|P(t, \gamma, r)|\left(1-u^{2}\right)^{\mu-1} d u \rightarrow 0
\end{aligned}
$$

as $r \rightarrow 1^{-}$provided that $t \neq \pm 1$ or $\mu \geqslant \frac{1}{2}+P$ with no restriction on $t$. By the same argument it follows that $\int_{0<\delta<\gamma \leqslant \pi}\left|G^{P}(x, t, r)\right| d t \rightarrow 0$ as $r \rightarrow 1^{-}$.

(b) From equations (3.5) and (3.7) we obtain

$$
\begin{aligned}
G^{P}(x, t, r)= & (-1)^{P} \frac{\mu}{\pi} \frac{(x-t)^{P}}{P !} \int_{-1}^{1}\left(1-r^{2}\right)\left(1-u^{2}\right)^{\mu-1} \\
& \cdot\left(\sum_{k=0}^{k<[P / 2]} \frac{a_{k}\left(1-t^{2}\right)^{\mu-1 / 2-k}}{\left(1-2 r \cos \gamma+r^{2}\right)^{\mu+1+P-k}}\left(\frac{\partial \cos \gamma}{\partial t}\right)^{P-2 k}\right. \\
& \left.+\frac{\left(1-t^{2}\right)^{\mu-1 / 2-P} A(t, \gamma, r)}{\left(1-2 r \cos \gamma+r^{2}\right)^{\mu+1+\eta}}\right) d u
\end{aligned}
$$

where $a_{k}, A(t, \gamma, r)$ and $\eta$ are defined in Lemma (3.3). We show that $\int_{-1}^{1}\left|G^{P}(x, t, r)\right| d t \leqslant M_{x}$ independent of $r$ by showing that each individual term in equation (3.8) has the same property. Indeed, by part (a), we need only show that 
the integral of each of these terms taken over $0 \leqslant \gamma \leqslant \delta$ is uniformly bounded. Let us write

$$
\begin{gathered}
G_{k}^{P}(x, t, r)=\frac{\mu}{\pi} \frac{(x-t)^{P}}{P !}\left(1-r^{2}\right) \int_{-1}^{1}\left(1-u^{2}\right)^{\mu-1} \frac{a_{k}\left(1-t^{2}\right)^{\mu-1 / 2-k}}{\left(1-2 r \cos \gamma+r^{2}\right)^{\mu+1+P-k}} \\
\cdot\left(\frac{\partial \cos \gamma}{\partial t}\right)^{P-2 k} d u, \quad 0 \leqslant k \leqslant\left[\frac{P}{2}\right] \\
F^{P}(x, t, r)=\frac{\mu}{\pi} \frac{(x-t)^{P}}{P !}\left(1-r^{2}\right) \int_{-1}^{1}\left(1-u^{2}\right)^{\mu-1} \frac{\left(1-t^{2}\right)^{\mu-1 / 2-P} A(t, \gamma, r)}{\left(1-2 r \cos \gamma+r^{2}\right)^{\mu+1+\eta}} d u .
\end{gathered}
$$

We make use of the well-known relation

$$
\frac{\mu}{\pi}\left(1-r^{2}\right) \int_{-1}^{1} \int_{-1}^{1} \frac{\left(1-t^{2}\right)^{\mu-1 / 2}\left(1-u^{2}\right)^{\mu-1}}{\left(1-2 r \cos \gamma+r^{2}\right)^{\mu+1}} d t d u=1
$$

and the fact that the integrand is positive. Relation (3.11) holds since the left-hand side is just

$$
\int_{-1}^{1} G^{0}(x, t, r) d t=\int_{-1}^{1} H(t, x, r) d t
$$

where $H(t, x, r)$ is the regular Abel kernel (see equation (3.3)). Now by equation (3.9) we obtain

$$
\begin{aligned}
\int_{0<\gamma<\delta}\left|G_{k}^{P}(x, t, r)\right| d t \leqslant & \frac{\mu}{\pi} \frac{\left(1-r^{2}\right)}{P !} \int_{0<\gamma<\delta} \int_{-1}^{1} \frac{\left(1-u^{2}\right)^{\mu-1}\left(1-t^{2}\right)^{\mu-1 / 2}}{\left(1-2 r \cos \gamma+r^{2}\right)^{\mu+1}} \\
& \cdot\left\{\frac{|x-t|^{P}\left|a_{k}\right|}{\left(1-t^{2}\right)^{k}\left(1-2 r \cos \gamma+r^{2}\right)^{P-k}}\left|\frac{\partial \cos \gamma}{\partial t}\right|^{P-2 k}\right\} d t d u .
\end{aligned}
$$

The proof will be completed if we show that the quantity in braces is bounded when $r \rightarrow 1$. Since $\left|a_{k}\right| /\left(1-t^{2}\right)^{k}$ is bounded for $t \in[a, b] \subset(-1,1)$ we investigate only the behavior of

$$
L(x, t, u, r)=\frac{|x-t|^{P}}{\left(1-2 r \cos \gamma+r^{2}\right)^{P-k}}\left|\frac{\partial \cos \gamma}{\partial t}\right|^{P-2 k}
$$

when $r \rightarrow 1$. As a function of $u, L(x, t, u, r)$ attains its maximum at $u=1$, i.e., $\cos \gamma=x t+\sqrt{\left(1-t^{2}\right)\left(1-x^{2}\right)}$. Expanding $\cos \gamma$ in terms of $(x-t)$ yields

$$
\cos \gamma-1=(x-t)^{2}[1+o(1)] \text { and } \frac{\partial \cos \gamma}{\partial t}=(x-t)[1+o(1)] \text {. }
$$

Hence, $\left(1-2 r \cos \gamma+r^{2}\right)^{P-k}=(x-t)^{2 P-2 k}[1+o(1)]$ as $r \rightarrow 1$ and

$$
L(x, t, r) \approx \frac{(x-t)^{P}(x-t)^{P-2 k}[1+o(1)]}{(x-t)^{2 P-2 k}[1+o(1)]}=O(1) \quad \text { as } r \rightarrow 1
$$


As for $F^{P}(x, t, r)$, we have

$$
F^{P}(x, t, r) \approx \frac{(x-t)^{P}}{(x-t)^{2 \eta}[1+o(1)]}=O(1) \quad \text { as } r \rightarrow 1
$$

since from Lemma (3.3), we have $\eta=(P-2) / 2,(P-1) / 2$ according to whether $P$ is even or odd.

(c) It is easy to see that $G^{0}(x, t, r) \rightarrow \delta(t-x)$ as $r \rightarrow 1$ (see [14]); then it follows from the relation $t^{P} \delta^{(P)}(t)=(-1)^{P} P ! \delta(t)$ that $G^{P}(x, t, r) \rightarrow \delta(t-x)$ as $r \rightarrow 1$. That is

$$
\lim _{r \rightarrow 1}\left\langle G^{P}(x, t, r), \phi(t)\right\rangle=\langle\delta(t-x), \phi(t)\rangle=\phi(x)
$$

for all $\phi \in C^{\infty}$, supp $\phi \subset(-1,1)$.

Take $\phi=1$ on $[a, b]$; then $\lim _{r \rightarrow 1}\left\langle G^{P}(x, t, r), \phi\right\rangle=1$, i.e.

$$
\lim _{r \rightarrow 1}\left(\int_{-1}^{a}+\int_{a}^{b}+\int_{b}^{1}\right) G^{P}(x, t, r) \phi(t) d t=1 .
$$

But from part (a), we immediately get

$$
\lim _{r \rightarrow 1} \int_{a}^{b} G^{P}(x, t, r) d t=1 \text {. Q.E.D. }
$$

COROllaRy. $G^{P}(x, t, r)$ is a quasi-positive kernel.

THEOREM (3.1). Let $f$ be a generalized function with support in $(-1,1)$ given by $f=\sum_{n=0}^{\infty} a_{n} C_{n}^{\mu}$. If $f$ has a value $\gamma$ at $\alpha \in(-1,1)$, then $\sum_{n=0}^{\infty} a_{n} C_{n}^{\mu}(\alpha)$ is Abel summable to $\gamma$.

Proof. By hypothesis there exists a nonnegative integer $P$ and a continuous function $F(t)$ such that

$$
F^{(P)}=f
$$

in a neighborhood of $\alpha$ and $\lim _{t \rightarrow \alpha} F(t) /(t-\alpha)^{P}=\gamma / P$ !. Without loss of generality, we may assume that (3.12) holds globally. Therefore,

$$
\begin{aligned}
\sum_{n=0}^{\infty} a_{n} C_{n}^{\mu}(\alpha) r^{n} & =\sum_{n=0}^{\infty} \frac{1}{h_{n}^{\mu}} C_{n}^{\mu}(\alpha) r^{n}\left(f,\left(1-t^{2}\right)^{\mu-1 / 2} C_{n}^{\mu}(t)\right) \\
& =\sum_{n=0}^{\infty} \frac{1}{h_{n}^{\mu}} C_{n}^{\mu}(\alpha) r^{n}\left(F^{P},\left(1-t^{2}\right)^{\mu-1 / 2} C_{n}^{\mu}(t)\right) \\
& =(-1)^{P} \sum_{n=0}^{\infty} \frac{1}{h_{n}^{\mu}} C_{n}^{\mu}(\alpha) r^{n} \int_{-1}^{1} F(t) \frac{d^{P}}{d t^{P}}\left(1-t^{2}\right)^{\mu-1 / 2} C_{n}^{\mu}(t) d t \\
& =\int_{-1}^{1}\left(\frac{F(t) P !}{(t-\alpha)^{P}}\right)\left(\frac{(-1)^{P}(t-\alpha)^{P}}{P !} \sum_{n=0}^{\infty} \frac{r^{n}}{h_{n}^{\mu}}\right. \\
& \left.=\int_{-1}^{1}\left(\frac{F(t) P !}{(t-\alpha)}\right)\left(\frac{d^{P}}{d t^{P}}\left(1-t^{2}\right)^{\mu-1 / 2} C_{n}^{\mu}(t)\right)\right) d t
\end{aligned}
$$

Applying Lemmas (3.4) and (3.1) finishes the proof. Q.E.D. 
ReMARKs. (i) If $P=0$, i.e., if $f(x)$ is a function in the classical sense for which the coefficients $\left\{a_{n}\right\}_{n=0}^{\infty}$ exist, we obtain the well-known result that the series $\sum_{n=0}^{\infty} a_{n} C_{n}^{\mu}(x)$ is Abel summable to $f(x)$ where $x$ is a point of continuity of $f(x)$ in $(-1,1)$.

(ii) It is natural to ask whether the converse of Theorem (3.1) is true. That is, if $\sum_{n=0}^{\infty} a_{n} C_{n}^{\mu}(\alpha)$ is Abel summable, does $\sum_{n=0}^{\infty} a_{n} C_{n}^{\mu}(x)$ have a value at $x=\alpha$ ? The answer is negative and $\delta^{\prime}(x)$ is a counterexample. For

$$
\delta^{\prime}(0)=\frac{\mu}{\pi} \sum_{n=0}^{\infty} \frac{1}{h_{n}^{\mu}} C_{n}^{\mu}(0) \frac{d}{d t} C_{n}^{\mu}(0)
$$

and $C_{n}^{\mu}(0)=0$ if $n$ is odd and $(d / d t) C_{n}^{\mu}(0)=0$ if $n$ is even. It is very interesting to find sufficient conditions for the converse; however, we will not pursue this here.

COROLlary. Under the same assumptions of the previous theorem if

$$
g(t)=\left(1-t^{2}\right)^{\mu-1 / 2} f(t),
$$

then $\lim _{\varepsilon \rightarrow 0}[\hat{g}(\alpha+i \varepsilon)-\hat{g}(\alpha-i \varepsilon)]=\left(1-\alpha^{2}\right)^{\mu-1 / 2} \gamma$.

Proof. From Theorem (2.1), we obtain $\hat{g}(z)=\sum_{n=0}^{\infty} a_{n} Q_{n}^{\mu}(z)$. Hence

$$
\hat{g}(\alpha+i \varepsilon)-\hat{g}(\alpha-i \varepsilon)=\sum_{n=0}^{\infty} a_{n} \int_{-1}^{1}\left(1-t^{2}\right)^{\mu-1 / 2} C_{n}^{\mu}(t)\left(\frac{2 i \varepsilon}{(t-\alpha)^{2}+\varepsilon^{2}}\right) d t .
$$

Taking the limit as $\varepsilon \rightarrow 0$, keeping in mind that the quantity in brackets is just the Poisson kernel for the upper half-plane and that the series $\sum_{n=0}^{\infty} a_{n} C_{n}^{\mu}(\alpha)$ is Abel summable to $\gamma$, we immediately get

$$
\lim _{\varepsilon \rightarrow 0}[\hat{g}(\alpha+i \varepsilon)-\hat{g}(\alpha-i \varepsilon)]=\left(1-\alpha^{2}\right)^{\mu-1 / 2} \gamma
$$

THEOREM (3.2). Let $f(x)$ be a generalized function with support in $(-1,1)$ given by the series $f(x)=\sum_{n=0}^{\infty} a_{n} C_{n}^{\mu}(x)$. Suppose that $f^{[\mu])}(x)=d^{[\mu]} f / d x^{[\mu]}$ has a value $\gamma$ at $\alpha \in(-1,1)$; then

$$
\phi(z)=\sum_{n=0}^{\infty} a_{n} h_{n}^{\mu} z^{n} \rightarrow \phi(\beta) \text { as } z \rightarrow \beta \text { radially, }
$$

where $\alpha=\frac{1}{2}(\beta+1 / \beta)$.

Proof. From the identity $\sum_{n=0}^{\infty} C_{n}^{\mu}(t) z^{n}=\left(1-2 t z+z^{2}\right)^{-\mu},|z|<1$, and the fact that $\left(1-t^{2}\right)^{\mu-1 / 2} /\left(1-2 t z+z^{2}\right)^{\mu}$ is in $C^{\infty}(I)$ where $I$ is a neighborhood of $\operatorname{supp} f$, we obtain

$$
\begin{aligned}
\left(f, \frac{\left(1-t^{2}\right)^{\mu-1 / 2}}{\left(1-2 t z+z^{2}\right)^{\mu}}\right) & =\sum_{n=0}^{\infty} a_{n}\left(C_{n}^{\mu}(t), \frac{\left(1-t^{2}\right)^{\mu-1 / 2}}{\left(1-2 t z+z^{2}\right)^{\mu}}\right) \\
& =\sum_{n=0}^{\infty} a_{n}\left(C_{n}^{\mu}(t),\left(1-t^{2}\right)^{\mu-1 / 2} \sum_{m=0}^{\infty} C_{m}^{\mu}(t) z^{m}\right) \\
& =\sum_{n=0}^{\infty} a_{n} \sum_{m=0}^{\infty} z^{m}\left(C_{n}^{\mu}(t),\left(1-t^{2}\right)^{\mu-1 / 2} C_{m}^{\mu}(t)\right) \\
& =\sum_{n=0}^{\infty} a_{n} \sum_{m=0}^{\infty} z^{m} h_{m}^{\mu} \delta_{n m}=\sum_{n=0}^{\infty} a_{n} h_{n}^{\mu} z^{n}=\phi(z), \quad|z|<1 .
\end{aligned}
$$


Since $f^{([\mu])}$ has a value $\gamma$ at $\alpha$, then there exists a continuous function $F$ such that $F^{(k)}=f^{([\mu])}$ in a neighborhood of $\alpha$ and $F(t) /(t-\alpha)^{k} \rightarrow \gamma / k !$ as $t \rightarrow \alpha$.

Without loss of generality we may assume that $k \geqslant[\mu]$ and $F^{(k)}=f^{([\mu)}$ globally where $F$ is continuous and has support in $(-1,1)$. Let $P=k-[\mu]$; then

$$
\begin{aligned}
\phi(z) & =\left(F^{(P)}, \frac{\left(1-t^{2}\right)^{\mu-1 / 2}}{\left(1-2 t z+z^{2}\right)^{\mu}}\right)=\left(F,(-1)^{P} \frac{d^{P}}{d t^{P}} \frac{\left(1-t^{2}\right)^{\mu-1 / 2}}{\left(1-2 t z+z^{2}\right)^{\mu}}\right) \\
& =\int_{-1}^{1} F(t)(-1)^{P} \frac{d^{P}}{d t^{P}} \frac{\left(1-t^{2}\right)^{\mu-1 / 2}}{\left(1-2 t z+z^{2}\right)^{\mu}} d t .
\end{aligned}
$$

As in the proof of Lemma (3.3) we can show that

$$
\frac{d^{P}}{d t^{P}} \frac{\left(1-t^{2}\right)^{\mu-1 / 2}}{\left(1-2 t z+z^{2}\right)^{\mu}}=\frac{\left(1-t^{2}\right)^{\mu-1 / 2-P} P(t, z)}{\left(1-2 t z+z^{2}\right)^{\mu+P}}
$$

where $P(t, z)$ is a polynomial in $t$ and $z$. Then, we have

$$
\begin{aligned}
\phi(z) & =\int_{-1}^{1} F(t) \frac{\left(1-t^{2}\right)^{\mu-1 / 2-P}}{\left(1-2 t z+z^{2}\right)^{\mu+P}} P(t, z) d t \\
& =\left(\int_{|t-\alpha|>\delta}+\int_{|t-\alpha|<\delta}\right) F(t) \frac{\left(1-t^{2}\right)^{\mu-1 / 2-P}}{\left(1-2 t z+z^{2}\right)^{\mu+P}} P(t, z) d t .
\end{aligned}
$$

Let us denote the first integral by $I(z)$ and the second by $J(z)$. Then,

$$
I(z)=\frac{1}{(-2 z)^{\mu+P}} \int_{|t-\alpha|>\delta} F(t) \frac{\left(1-t^{2}\right)^{\mu-1 / 2-P}}{\left[t-\frac{1}{2}(1 / z+z)\right]^{\mu+P}} P(t, z) d t
$$

and by choosing $z$ sufficiently close to $\beta$ we can make $\left|\frac{1}{2}(1 / z+z)-\alpha\right|<\delta / 2$. Therefore,

$$
|I(z)| \leqslant \int_{|t-\alpha|>\delta}|F(t)| \frac{\left(1-t^{2}\right)^{\mu-1 / 2-P}}{(\delta / 2)^{\mu+P}}|g(t)| d t
$$

where

$$
g(t)=\sup _{0<r<z<1}\left|P(t, z) /(-2 z)^{\mu+P}\right|, \quad g \in L^{1}[-1,1],
$$

and by the Lebesgue dominated convergence theorem it follows that $\lim _{z \rightarrow \beta} I(z)$ exists.

As for $J(z)$ we have

$$
\begin{aligned}
|J(z)| & \leqslant \int_{|t-\alpha|<\delta}|F(t)| \frac{\left(1-t^{2}\right)^{\mu-1 / 2-P}}{|t-\alpha|^{\mu+P}}|g(t)| d t \\
& =\int_{|t-\alpha|<\delta} \frac{|F(t)|}{|t-\alpha|^{P+[\mu]}} \frac{|t-\alpha|^{P+[\mu]}\left(1-t^{2}\right)^{\mu-1 / 2-P}}{|t-\alpha|^{\mu+P}}|g(t)| d t \\
& =\int_{|t-\alpha|<\delta} \frac{|F(t)|}{|t-\alpha|^{P+[\mu]}} \frac{\left(1-t^{2}\right)^{\mu-1 / 2-P}|g(t)|}{|t-\alpha|^{\mu-[\mu]}} d t .
\end{aligned}
$$


The last integral is bounded since $|F(t)| /|t-\alpha|^{P+[\mu]}$ is bounded and

$$
\frac{\left(1-t^{2}\right)^{\mu-1 / 2-P}|g(t)|}{|t-\alpha|^{\mu-[\mu]}}
$$

is integrable in a $\delta$-neighborhood of $\alpha$. Hence, applying Lebesgue dominated convergence once more yields that $\lim _{z \rightarrow \beta} J(z)$ exists. Q.E.D.

ACKnowledgement. The author wishes to thank Professor Gilbert Walter for considerable encouragement; this work developed out of a thesis written under his supervision.

\section{REFERENCES}

1. H. Bremermann, Distribution, complex variables, and Fourier transforms, Addison-Wesley, Reading, Mass., 1965.

2. A. Erdélyi, Higher transcendental functions. Vols. I, II, McGraw-Hill, New York, 1953-1955.

3. R. P. Gilbert, Constructive methods for elliptic equations, Lecture Notes in Math., vol. 365, Springer-Verlag, Berlin and New York, 1974.

4. __ Function theoretic methods in partial differential equations, Academic Press, New York, 1969.

5. __ Bergman's integral operator method in generalized axially symmetric potential theory, J. Math. Phys. 5 (1964), 983-997.

6. Integral operator methods in bi-axially symmetric potential theory, Contributions to Differential Equations 2 (1963), 441-456.

7. R. P. Gilbert and H. C. Howard, On the singularities of Sturm-Liouville expansions, Proc. Sympos. Analyt. Methods in Math. Phys. 8 (1967), 141-148.

8. __ A generalization of a theorem of Nehari, Bull. Amer. Math. Soc. 72 (1966), 37-39.

9. J. Hadamard, Théorème sur les séries entières, Acta Math. 22 (1898), 55-64.

10. S. Lojasiewicz, Sur la valeur et la limite d'une distribution dans un point, Studia Math. 16 (1957), $1-36$.

11. Z. Nehari, On singularities of Legendre expansions, Arch. Rational Mech. Anal. 5 (1956), 987-992.

12. G. Szegö, Orthogonal polynomials, Amer. Math. Soc. Colloq. Publ., vol. 23, Amer. Math. Soc., Providence, R. I., 1959.

13. G. Walter, On real singularities of Legendre expansions, Proc. Amer. Math. Soc. 19 (1968), 1407-1412.

14. __ Expansion of distributions, Trans. Amer. Math. Soc. 116 (1965), 492-510.

15. A. Zygmund, Trigonometric series, Vol. 1, Cambridge Univ. Press, London, 1959.

Department of Mathematics, University of Wisconsin, Milwaukee, Wisconsin 53201

Current address: Department of Mathematics, California Polytechnic State University, San Luis Obispo, California 93407 